12 Dazu Neubacher, Kriminologische Grundlagen einer internationalen Strafgerichtsbarkeit, 2005; Ahlbrecht, Geschichte der völkerrechtlichen Strafgerichtsbarkeit im 20. Jahrhundert, 1999.

13 Jäger, Makrokriminalität, S. 13; ähnl. Jäger, Versuch über Makrokriminalität, in: Strafverteidiger 1988, S. 172, 175.

14 Vgl. Neubacher, Politik und Verbrechen - Zur Terminologie und Typologie staatlicher bzw. gegen den Staat gerichteter Kriminalität, in: MschrKrim 2002, S. 290 ff.

15 Weiter ausgeführt in: Neubacher, Tribunal der Vernunft oder hilfloser Versuch einer Verrechtlichung? - Acht Thesen zum ständigen Internationalen Strafgerichtshof, in: Recht und Politik 2003, S. $160 \mathrm{ff}$.

16 Siehe H.J. Schneider, Der gegenwärtige Stand der Opferforschung, in: MschrKrim 1998, S. 327; Orth, Strafgerechtigkeit und Bewältigung krimineller Viktimisierung, 2001, S. 19 f.

17 Vgl. Davison/Neale, Klinische Psychologie, 3. Aufl. 1988, S. 192; Yule (ed.), Post-Traumatic Stress Disorders: Concepts and Therapy, 1999.

18 H.J. Schneider, MschrKrim 1998, S. 329.

$19 \mathrm{Vgl}$. Kilchling, Opferinteressen und Strafverfolgung, 1995, S. 630, 673; Baurmann/Schädler, Das Opfer nach der Straftat - seine Erwartungen und Perspektiven, 1991, S. 279; H.J. Schneider, MschrKrim 1998, S. 333.

20 Kiefl/Lamnek, Soziologie des Opfers, 1986, S. 239 ff.; H.J. Schneider, MschrKrim 1998, S. 329.

21 Orth, Strafgerechtigkeit und Bewältigung krimineller Viktimisierung, 2001, S. 126.

22 Vgl. Ambos, Straflosigkeit von Menschenrechtsverletzungen, Zur »impunidad « in südamerikanischen Ländern aus völkerstrafrechtlicher Sicht, 1997.

23 Siehe auch Jäger, Verbrechen unter totalitärer Herrschaft, in: Handwörterbuch der Kriminologie, 2. Aufl. 1975, Dritter Band, S. $458 \mathrm{ff}$.

24 Zit. nach Cassesse, On the Current Trends towards Criminal Prosecution and Punishment of Breaches of International Humanitarian Law, in: European Journal of International Law 1998, S. 6.

25 Dazu Möller, Völkerstrafrecht und Internationaler Strafgerichtshof, 2003, S. $548 \mathrm{ff}$.

26 Werle, Völkerstrafrecht, 2003, S. 71 ff., 76; Nerlich, Apartheidkriminalität vor Gericht, 2002, S. 1 f. Ob eine solche Verpflichtung auch Drittstaaten trifft, ist im Einzelnen noch ungeklärt.
$27 \mathrm{Vgl}$. Sellin/Wolfgang, The Measurement of Delinquency, 1964, S. 278 Evans/Scott, The Seriousness of Crime Cross-Culturally, in: Criminology 1984, S. 41, 50; Kania/Brand/Zimmermann/Walter, Die Einschätzung von Gewaltdelikten im europäischen Vergleich, in: MschrKrim 2003, S. 247 ff

$28 \mathrm{Vgl}$. Nemitz, Strafzumessung im Völkerstrafrecht, 2002, S. 165.

29 So auch Reemtsma, Das Recht des Opfers auf die Bestrafung des Täters - als Problem, 1999, S. 25 f.

$30 \mathrm{Vgl}$. Frei, Vergangenheitspolitik, Die Anfänge der Bundesrepublik und die NS-Vergangenheit, 1999, S. 129, 229.
31 So Schreiber, Deutsche Kriegsverbrechen in Italien. Täter, Opfer, Strafverfolgung, 1996, S. 48 m.w.N

32 Jäger, Verbrechen unter totalitärer Herrschaft, 1967, S. 94 ff., 120 ff.

33 Browning, Ganz normale Männer, Das Reserve-Polizeibataillon 101 und die »Endlösung « in Polen, 1999, S. 87 f., 98 ff., s. für das Völkerstrafrecht auch Ambos, Der Allgemeine Teil des Völkerstrafrechts, 2002, S. 863.

34 Jäger, Versuch über Makrokriminalität, in: Strafverteidiger 1988, S. 177.

35 Vgl. Neubacher/Walter, Sozialpsychologische Experimente in der Kriminologie, 2002.

36 Mit Beispielen (u.a. aus Chile, Guatemala, El Salvador) und weiteren Nachweisen s. Neubacher, Kriminologische Grundlagen einer internationalen Strafgerichtsbarkeit, 2005, S. $456 \mathrm{ff}$

37 Nerlich, Apartheidkriminalität vo Gericht, Der Beitrag der südafrikanischen Strafjustiz zur Aufarbeitung von Apartheidunrecht, 2002, S. 335

$38 \mathrm{Vgl}$. Kutz, Amnestie für politische Straftäter in Südafrika, Von der Sharpeville-Amnestie bis zu den Verfahren der Wahrheits- und Versöhnungskommission, 2001, S. 100.

\title{
Perspektiven für den deutschen Strafvollzug
}

\section{Bernd Maelicke}

\section{Veränderte gesellschafts- und sozialpolitische Rahmenbedingungen}

ie grundlegenden und rapiden Veränderungen in Staat und Gesellschaft wirken sich selbstverständlich auch auf den deutschen Strafvollzug aus:

Die Globalisierung führt zu Umbrüchen in einem bisher unbekannten und unerwarteten Ausmass. Innovation und Modernisierung erfassen alle gesellschaftlichen Bereiche. Der Wegfall der ideologischen und nationalen Grenzen führt zur totalen Verunsicherung. Prinzipiell alle bisher tragenden und unbestrittenen Werte und Normen sind in Frage gestellt. »Global Denken« ermöglicht sowohl Fortschritt wie Rückschritt - Orientierung an humanen Grundwerten und sozialen Mindeststandards, aber auch Übernahme inhumaner Zielvorstellungen und Neudefinition von Armut und verstärkter Ausgrenzung. »Lokal Handeln« ermöglicht Eingehen auf regionale und situationsspezifische Besonderheiten, bedeutet aber auch den zunehmenden Verzicht auf überregionale Wertorientierungen und verbindliche Mindeststandards.

Der weltweite Wettbewerb der Betriebe und Unternehmen ergreift auch die Staaten und Gesellschaften.
Gewinnorientierung und Wirtschaftlichkeit bestimmen mehr und mehr das Handeln und das Bewusstsein der Mitarbeiter und des Managements - in der Wirtschaft, bei staatlichen Organisationen, ja sogar bei Non-Profit-Organisationen. Effektivität und Effizienz, verbunden mit wirksamen betriebswirtschaftlichen Methoden, werden zu den dominanten Erfolgsfaktoren.

Der deutsche Sozialstaat befindet sich in einer strukturellen und nahezu unlösbaren Finanzkrise. Die deutsche Einheit, fehlendes Wachstum und die strukturelle Arbeitslosigkeit haben alle Sozialleistungssysteme an die Grenze der Insolvenz geführt. Die demographische Entwicklung führt zu weiteren Belastungen, für die weder Politik noch Wissenschaft überzeugende Lösungen anbieten können.

\section{Veränderte kriminalpoliti- sche Rahmenbedingungen}

Der Umbruch in Staat und Gesellschaft erfasst selbstverständlich auch die Kriminalpolitik. Verunsicherte Bürger und aggressive Medien setzen die Politik so unter Druck, dass ein regelrechter Wettbewerb darüber stattfindet, wer mehr Sicherheit versprechen kann - unabhängig davon, ob diese Versprechen einge- löst werden können und möglicherweise zu noch mehr Verunsicherung führen.

Das in den 70er und 80er Jahren vorhandene Reform-Bündnis zwischen der Praxis, der Wissenschaft, dem Gesetzgeber, der Rechtsprechung, den Medien und rationalen Kriminalpolitikern ist zerbrochen. Aggressive Medien und populistische und opportunistische Politiker bestimmen die öffentliche Diskussion und das Handeln. Fach- und Führungskräfte fühlen sich verunsichert und verlassen, fachliche Mindeststandards der Resozialisierung geraten ins Wanken.

Wegfall der Grenzen und verschärfte Gesetze führen zur Geissel der Überlegung in den Anstalten menschenunwürdige Unterbringung überlagert alle gutgemeinten Behandlungsabsichten. Weitere Frustration von Mitarbeitern und Gefangenen ist die Folge. Die Öffentlichkeit gewöhnt sich an Zustände, die grundgesetzwidrig sind.

\section{Wirkungsorientierte Steuerung}

Unabhängig von der geschilderten Umbruchsituation und bereits seit Jahrzehnten wissenschaftlich und in vielen Kommissionen vorbereitet gewinnt in der Fachdiskussion und in der Praxis das fachliche Ziel einer verstärkten Wirkungsorientierung in der ambulanten und stationären Resozialisierung immer mehr an Bedeutung. Welche Behandlungsziele können durch welche Methoden mit welchem Wirkungsgrad reali- 
siert werden ? Was bedeuten jeweils im Einzelfall und für die gesamte Dienstleistungs-Organisation fachliche Effektivität und betriebswirtschaftliche Effizienz? Kosten- und Leistungsrechnung, Qualitätsmanagement, Budgetierung, Controlling sind nur einige Beispiele der Instrumente der Strategie und Konzeption des "New Public Management", das bundesweit in allen Diensten und Einrichtungen in öffentlicher und privater Trägerschaft eingeführt wird.

»Outsourcing « gehört ebenso zu diesem Programm - also die Übertragung von Aufgaben auf leistungsfähige und leistungsbereite Dritte. Im Vollzug werden in nahezu allen Bundesländern immer mehr derartiger Projekte realisiert. Die JVA Neumünster in Schleswig-Holstein und die teilprivatisierte JVA Hünfeld in Hessen sind zwei gute Beispiele für Quantität und Qualität dieses Ansatzes.

Das Paradigma der »Neuen Steuerung « stellt völlig neue Anforderungen an die Vollzugsorganisation und das Vollzugsmanagement. Die neuen Aufgaben erhöhen die Bedeutung des Behandlungs- und Sicherheitsmanagements auf den Abteilungen, d.h. insbesondere der Vollzugsabteilungsleiter und ihrer Stationsbeamten des Allgemeinen Vollzugsdienstes. Die Aufgaben des TOP-Managements (Anstaltsleiter, stellv. Anstaltsleiter und Verwaltungs-Dienstleiter) sind im Sinne eines A-B-C-Modells neu zu definieren und zu justieren. Behandlungs- wie Sicherheitsmanagement benötigen eine permanente Fortentwicklung ihrer Prozessqualität und ein entsprechend stark besetztes Controlling. Gleiches gilt für die Entwicklung und Steuerung des Outsourcing-Konzeptes in den Anstalten.

\section{Verantwortbare Haftvermei- dung und Haftverkürzung}

Nicht nur wegen der Geissel der Überbelegung muss aus konzeptionellen und fachlichen Gründen an der Entwicklung und Realisierung eines Gesamtkonzeptes der ambulanten und stationären Resozialisierung festgehalten werden. Behandlung und Wiedereingliederung ist ein viele Jahre anhaltender Prozess, der im
Sinne eines professionellen case-managements von den zuständigen Fachkräften der zahlreichen beteiligten Organisationen im Rahmen eines Gesamtplanes in jedem Einzelfall zu steuern ist.

Kriminologisch ist unbestritten, dass Resozialisierungserfolge nur in der Freiheit und nicht hinter Mauern wirksam und dauerhaft festgestellt werden können. Ambulante Massnahmen (als Alternativen oder nach der Entlassung) haben deshalb eine hohe Relevanz - Vollzugsschäden werden durch sie vermieden, die soziale Integration wird erleichtert, weitere Ausgrenzung verhindert. $\mathrm{Zu}-$ dem sind sie extrem kostengünstiger.

Sie entlasten quantitativ und qualitativ den Vollzug, vermeiden »Fehlbelegung ", haben geringere Rückfallquoten. Sie ermöglichen für die »Restgruppe« der Strafgefangenen einen zielgruppenbezogenen Behandlungsvollzug mit verbesserten Handlungsmöglichkeiten und gesteigerter Wirksamkeit (bezogen auf Behandlungsprogramme und auf unverzichtbare Sicherheitsstandards).

Die beigefügte Übersicht »10 Schritte der kriminalpolitischen Feldentwicklung nach der Methode der offenen Koordination und Kooperation« zeigen am Beispiel des Landes Schleswig-Holstein, wie dort in den Jahren 1990 bis 2005 eine derartige Programmatik realisiert worden ist.

\section{Soziale Integration oder Ausgrenzung?}

Die letzten 30 Jahre wurden durch ein Resozialisierungskonzept im Sinne einer Sozialen Strafrechtspflege bestimmt, dass sich sowohl in der Rechtsprechung des BVerfG wie in den einschlägigen bundesgesetzlichen Regelungen durchgesetzt hat - jeweils mit entsprechenden Mehrheiten in Bundestag und Bundesrat.

Dieser Konsens wurde nun durch verschiedene Gesetzesinitiativen gezielt aufgekündigt - Reduzierung der Behandlungsprogrammatik, Verschärfung der Strafrahmen und vorrangige Betonung des Sicherheitsgedankens sind die Reaktionen von unter Druck gesetzten Politikern und Parteien auf die aggressive Medienberichterstattung über spektakuläre Einzelfälle. Der Geist der Irrationalität ist aus der Flasche, die "gefühlte Sicherheit »der Bevölkerung ist ein neuer Maßstab auch für eine rationale Gesellschafts- und Kriminalpolitik.

Hier rächt sich eine programmatische und strukturelle Vernachlässigung von Opferinteressen im Konzept der Sozialen Strafrechtspflege, die dringend aufgehoben werden muss - ohne die Richtigkeit des Resozialisierungskonzepts und der Täterorientierung aufzugeben. Aber es zeigt sich auch, wie schnell marktund wettbewerbsorientierte Medien und opportunistische Politiker bereit sind, mit - berechtigten wie unberechtigten Ängsten - ihre Geschäfte zu machen.

Jeder Straftäter, auch der Sexualund Gewalttäter, hat den Anspruch auf die Perspektive der Resozialisierung, der Entlassung, der Wiedereingliederung. Ausgrenzung durch Freiheitsentziehung muss ultima ratio bleiben, darf nicht Mittel zum
Zweck der Behauptung von politischer Handlungsfähigkeit oder der Steigerung von Verkaufsauflagen werden.

Und da wo Behandlungsfähigkeit und -willigkeit nicht festgestellt werden können, dürfen humane Mindeststandards auch bei einem Höchstmaß an Sicherheit nicht vernachlässigt werden. »Wegsperren " reicht nie als Antwort im Sinne des Grundgesetzes aus.

\section{Der deutsche Strafvollzug am Scheideweg}

Es ist deutlich, dass eine RichtungsVergewisserung erforderlich ist:

Bekennt sich die deutsche Gesellschaft zu ihrem im Grundgesetz verankerten Menschenbild, das im Konzept der Sozialen Strafrechtspflege und im »Angebots- und Chancenvollzug « seinen Niederschlag findet? Nehmen Politiker, Parteien und Medien ihre daraus resultierende aufklärende und integrierende Verantwortung wahr?

\section{Verantwortbare Haftvermeidung/Haftverkürzung}

10 Schritte der kriminalpolitischen Feldentwicklung nach der Methode der offenen Koordination und Kooperation

\section{Externe Begutachtung \\ - des Systems des Strafvollzugs \\ - der Sozialen Dienste der Justiz \\ - der Freien Straffälligenhilfe \\ - der externen Dienstleister}

\section{Befragung von}

- Fach- und Führungskräften von 1.

- von Richtern und Staatsanwälten

- von Rechtsanwälten, e.a. Mitarbeitern, Angehörigen

- von Betroffenen

zur Ist-Analyse und zu Vorschlägen und Empfehlungen

\section{Masterplan zur Umsetzung und Feldentwicklung}

4. Bildung von Projektgruppen und Fachkommissionen

5. Koordinierte Programmentwicklung für Aus- und Fortbildung der Fach- und Führungskräfte, Richter und Staatsanwälte etc.

\section{Koordinierte Personalentwicklung}

7. Gestaltung der rechtlichen, finanziellen und organisatorischen Rahmenbedingungen

8. Exemplarische Projektentwicklung

9. Controlling und Fortschreibung

10. Öffentlichkeitsarbeit, Marketing 
Haben auf Bundes- und Länderebene die zuständigen Politiker, Experten und Fach- und Führungskräfte die Kraft, an der Realisierung des fachlich unbestrittenen Konzepts der ambulanten und stationären Resozialierung (mit gleichwertiger Opferperspektive) festzuhalten, es fortzuentwickeln und die dafür notwendigen rechtlichen, materiellen und politischen Rahmenbedingungen zu gewährleisten?

Oder wird angestrebt bzw. billigend in Kauf genommen, dass Abstand genommen wird vom Konzept der sozialen Integration, dass (vermeintliche) Sicherheit mit der Folge der Ausgrenzung und Stigmatisierung das neue Leitbild wird? Die Bei- spiele in den USA und in Russland zeigen die Folgen: explodierende Gefangenenzahlen, steigende Kosten, kein Rückgang der Kriminalität., Angst vor den im Vollzug nur verwahrten und zur Entlassung anstehenden Gefangenen.

Die Diskussion über die Beibehaltung der Bundeszuständigkeit für die Gesetzgebung für den Strafvollzug oder über ihre Verlagerung auf die Länder wird eine Nagelprobe für diese Richtungsentscheidung werden: bleibt es bei der gesamtgesellschaftlichen Verantwortung und bei einer fachlichen Integration in ein Gesamtkonzept der Strafrechtspflege (mit einer Bundeszuständigkeit für StGb, StPO, JGG, KJHG, StVollzG, U-
HaftVollzG, JugendVollzG etc) oder wird diese Schwerpunktsetzung und fachliche und systematische Integration aufgegeben und 16 verschiedenen Länderregelungen überlassen?

Die Schritte der kriminalpolitischen Feldentwicklung sind noch lange nicht am Ziel angekommen, werden es nie sein, müssen ständig modifiziert und fortentwickelt werden. Rückschläge und Fehlentwicklungen sind nicht zu vermeiden dürfen aber nicht zur Orientierungslosigkeit oder zu populistischen Anpassungen verleiten. Die »Wirkungsorientierte Steuerung « wird mehr und mehr Effizienz und Effektivität ermöglichen.
Wo aber sind die opinion-leader die ihre gesamtgesellschaftliche Verantwortung rational wahrnehmen und Geduld und Stärke mitbringen, diese schwierigen Innovations- und Modernisierungsprozesse abzusichern?

Perspektiven für den deutschen Strafvollzug: mühsame Feldentwicklung in kleinen Schritten oder Ausgrenzungs-Strategie nach amerikanischem Muster?

Bernd Maelicke gehört zu den Heraugebern dieser Zeitschrift und lehrt als Hochschullehrer an der Universität Lüneburg

\section{Helga Einsele und ihre kriminalpolitischen Wirkungen in der Öffentlichkeit}

Bernd Maelicke

\begin{abstract}
n NK 2-2005 hat Hannelore Maelicke einen Nachruf auf Helga Einsele geschrieben. Dieser Beitrag wurde im Oktober als Vortrag auf dem 30jährigen Jubiläum des Mutter-KindHeimes der JVA Frankfurt des Vereins für Kinder von Trennungswaisen gehalten.
\end{abstract}

Wenn man die letzten 50 Jahre überblickt, so gehört Helga Einsele zu den wenigen Persönlichkeiten, die in der Öffentlichkeit positiv mit dem Thema Strafvollzug in Verbindung gebracht wurden und dies auch weiterhin werden. Sie hat sich ein Leben lang mit dem Programm eines »Strafvollzugs der positiven $\mathrm{Zu}$ wendung « identifiziert und zu Wort gemeldet - und sie fand in den Medien und bei den Bürgern überwiegend Verständnis und Unterstützung. Was sind die Gründe dafür was können wir von Helga Einsele lernen?

Bei einer solchen Fragestellung muss deutlich getrennt werden zwischen dem Einzigartigen und Persönlichkeitsspezifischen, dass sich mit dem Lebensweg und den Wirkungen von Helga Einsele verbindet und dem Verallgemeinerbaren, Über- tragbaren und Erlernbaren, das als Richtschnur für andere - professionell oder ehrenamtlich Tätige - gelten kann. Als Person ist Helga Einsele ist nur erklärbar durch Verlauf und Historie des 20. Jahrhunderts, das sie nahezu vollständig erlebt und erlitten hat, durch ihr Elternhaus, ihre Erfahrung als Mädchen in Jungenschulen, ihre Begegnung mit Gustav Radbruch, ihre »neuen Erfahrungen « in den USA, durch das Berufsverbot im Dritten Reich, ihre »Lebensentscheidung « für den Frauenvollzug, ihr lebenslanges politisches Engagement, ihre wissenschaftlichen und publizistischen Tätigkeiten. In ihrer vergriffenen Biografie "Mein Leben mit Frauen in Haft « - sie selbst bezeichnet dieses eindrucksvolle Werk als "Niederschrift « (hier ist deutlich die Handschrift ihrer Tochter Nele Löw-Beer zu erkennen) - und in der Festschrift zu ihrem 80. Geburtstag wird deutlich, dass hier eine Frau über viele Jahrzehnte hinweg Handlungsmaximen und humane Maßstäbe gesucht, gelebt und gesetzt hat, die für nahezu alle ihre Mitstreiterinnen und Mitstreiter hohe, teilweise allzu hohe Anforderungen darstellen. Dies wurde auch ein Pro- blem für einige ihrer Nachfolger im Amt der Gefängnisdirektorin.

Am 4. November 1947 begannen für Helga Einsele »fast 28 Jahre einer totalen Inanspruchnahme durch den Beruf mit den oft erschütternden Schicksalen von Frauen « und wie sie es lapidar ausdrückte »durch eine Reihe von meiner Meinung nach dazugehörenden politischen Nebentätigkeiten«. Ähnlich wie Radbruch nannte sie dieses Leben ein »entpersönlichendes Klosterleben « - aber, wie sie anfügt »vor allem geformt durch die Beziehung zu einer im hohen Maß verpflichtenden und in enger Verbindung mit nahe stehenden Mitarbeiterinnen zu erfüllenden Aufgabe«.

Übrig blieb damals (im Jahr 1947) »das Heimweh nach dem Gefährten in Österreich und eine vom schlechten Gewissen überschattete Verbundenheit mit einem kleinen Mädchen, das mehr und mehr zur Partnerin wurde « - und dies bis zu ihrem Tod im begnadeten Alter von 94 Jahren. Wie bereits erwähnt, ist diese partnerschaftliche Mitwirkung unter anderem deutlich nachzuverfolgen in den zahlreichen Veröffentlichun- gen von Helga Einsele, an denen Frau Nele Löw-Beer hilfreich unterstützend mitgewirkt hat.

Ihr »Leben um der Überzeugung willen «, ihr »Schwimmen gegen den Strom «, ihre reformerischen Aktivitäten in der Vollzugsanstalt für Frauen - all dies ist immer wieder beschrieben und gewürdigt worden und soll deshalb an dieser Stelle nicht wiederholt werden. Ihre Wirkungen halten auch 30 Jahren nach dem Ausscheiden aus dem aktiven Dienst immer noch an - ihre konzeptionellen und vollzugspolitischen Vorstellungen und Forderungen sind noch lange nicht erfüllt! Und erst recht nicht ihr Ruf und der ihres Lehrers Radbruch nach etwas Besserem als dem Strafrecht oder nach einer strukturellen Reduzierung der Zahl der U- und Strafgefangenen bei gleichzeitigem Ausbau von ambulanten Behandlungs- und Wiedereingliederungsangeboten.

Viele ihrer Ideen und Projekte sind sogar gefährdet - so sind z.B. die Zahlen des offenen Vollzuges und der vorzeitigen Entlassung rückläufig, Personal wird abgebaut, für ambulante Dienste und Nachsorgeein- 